Gerontologische Themen sind zunehmend häufiger auch Gegenstand oder Thema in Kulturformen wie Film, Fernsehen, Literatur etc. In dieser Rubrik möchten wir Ihnen immer wieder solche Beispiele vorstellen und sie aus gerontologischer Sichtweise interpretieren.

Kennen Sie selbst ein interessantes oder kontroverses Beispiel? Melden Sie sich gerne bei der Redaktion: gerontologie@sgg-ssg.ch.Stichwort:KULTUR.
De plus en plus fréquemment les thèmes gérontologiques font l'objet ou sont le sujet de formes culturelles comme les films, la télévision, la littérature etc. Dans cette rubrique, nous souhaitons vous en présenter des exemples et les interpréter d'un point de vue gérontologique.

Vous connaissez vous-même un exemple intéressant ou controversé ? N'hésitez pas à contacter la rédaction : gerontologie@sgg-ssg.ch. Mot clé : CULTURE.

\title{
Der alte Mann und sein Roboter
}

\author{
Kurt Seifert
}

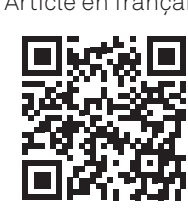

www.hgf.io

Der Film Robot \& Frank handelt vom Verhältnis zwischen Mensch und Maschine und dreht sich um die Frage, ob die virtuelle Welt einen Ersatz für das bieten kann, was Menschen brauchen: Beziehungen, die Sinn vermitteln.

\section{Le vieil homme et son robot}

Le film Robot \& Frank traite de la relation entre l'homme et la machine et tourne autour de la question de savoir si le monde virtuel peut remplacer ce dont les hommes ont besoin : des relations qui transmettent un message.

DOI 10.1024/2297-5160/a000035

\section{Der Hintergrund}

Wird die von Menschen konstruierte und programmierte künstliche Intelligenz sich bereits in nächster Zukunft als dem menschlichen Wissen und Können überlegen erweisen? Dieser Gedanke beschäftigt uns seit geraumer Zeit und er schlägt sich auch in Produkten der Massenkultur nieder. Hoffnungen wie Ängste sind damit verbunden: Künstliche Intelligenz könnte uns helfen, schneller ins «Reich der Freiheit» (Karl Marx) zu gelangen, indem wir

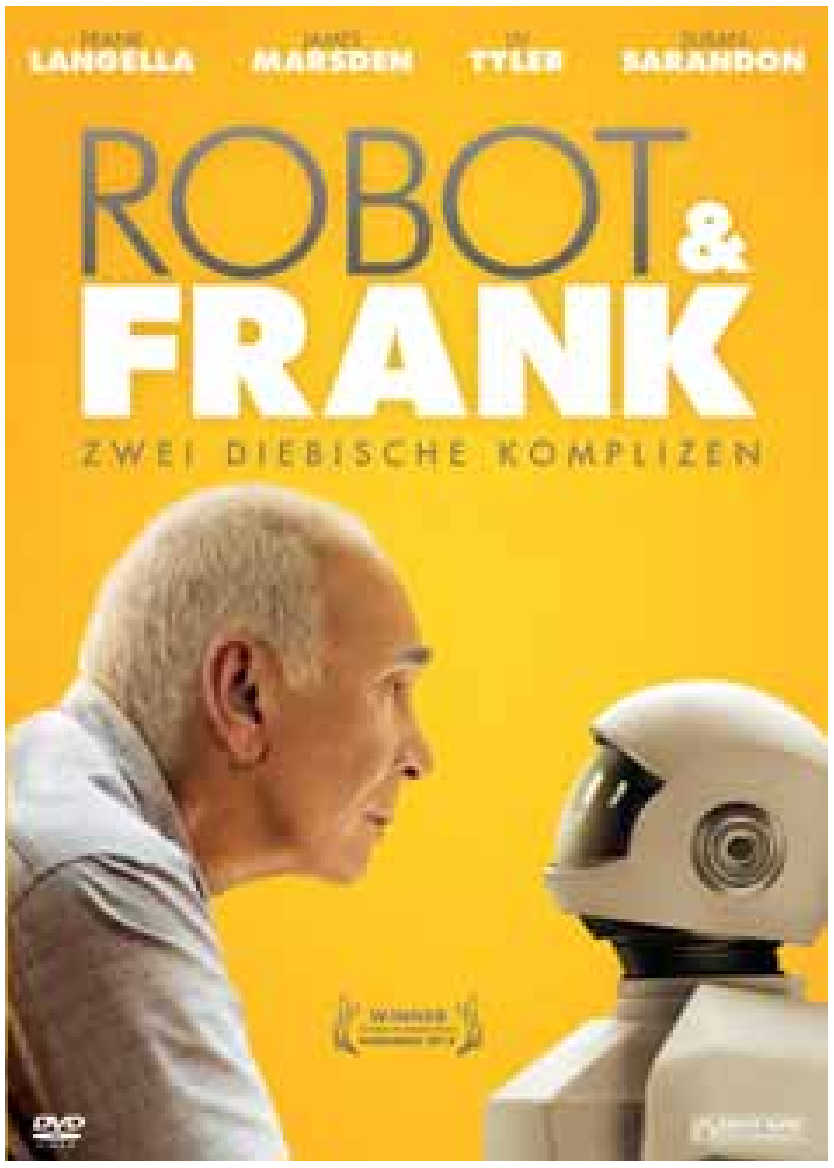

Robot \& Frank (2012), 86 Minuten, Senator Home Entertainment. Drehbuch: Christopher Ford, Regie: Jake Schreier. 
Roboter für uns arbeiten lassen. Sie könnte allerdings auch in die absolute Abhängigkeit von einer von uns selbst geschaffenen Welt führen. Die heutige Kulturindustrie schafft massenhaft solche negativen Utopien.

\section{Der Film}

Die Maschine als Helferin oder als Herrscherin über den Menschen ist ein Fragenfeld, dem sich der Film Robot $d \sigma$ Frank recht locker nähert - und eine eigenwillige Antwort zu geben versucht. Frank (gespielt vom sehr glaubwürdig wirkenden Frank Langella) ist ein ziemlich vergesslicher alter Mann, der in seinen besten Jahren ein bekannter Juwelendieb war. Sein Sohn Hunter (James Marsden), von dem man nicht erfährt, was er sonst so treibt, macht sich Sorgen um den Vater und möchte ihn in einem Heim unterbringen. Frank bleibt eigensinnig auch nachdem ihm der Sohn einen Playmobil-ähnlichen Pflegeroboter in Menschengrösse aufdrängt. Dieser soll ihn zu einer gesunden Ernährung sowie zu einem strukturierten Tagesplan mit Spaziergängen und Gartenarbeit anhalten.

Das wirkt an dieser Stelle wie eine leichte Parodie auf mancherlei «Aktivierungsmodelle», die in Alterseinrichtungen gang und gäbe sind. Doch Frank lässt sich durch seinen Roboter nicht zu sinnvoller Tätigkeit motivieren. Er gewinnt aber auf andere Weise Freude am neuen Hausbewohner: Indem er ihn zum Komplizen für seine Einbruchspläne macht. Der alte Ganove kann's nämlich nicht lassen - und so vergesslich ist er noch nicht, um seine früheren Fähigkeiten nicht reaktivieren zu können. Der Roboter scheint zunächst zu zögern, doch dann willigt er ein, sich zum Tatwerkzeug machen zu lassen. Er ist allerdings mehr als ein Werkzeug, scheint menschenähnliche Züge tragen - obwohl der Roboter mehrfach betont, er sei keine Person, sondern lediglich ein Programm.

Die Polizei kommt Frank aufgrund von Computerrecherchen auf die Spur und, um sich selbst nicht zu belasten, muss er schweren Herzens das «Gedächtnis» seines Komplizen löschen und so den Roboter deaktivieren denn dieser sei ihm zum «Freund» geworden, wie Frank seinem Sohn Hunter gesteht. Das künstliche Wesen ist also weder Herr noch Knecht, sondern so etwas wie ein Gegenüber. Für einen Moment lang scheint die Humanisierung der Technik möglich geworden zu sein.

\section{Die Interpretation}

Die Geschichte des Films spielt in einer «nahen Zukunft», wie zu Beginn der Handlung eingeblendet wird. Das Geschehen führt jedoch nicht in eine bessere Welt hinaus, sondern in eine, die menschliche Begegnung durch ausgefeilte Technik zu ersetzen sucht. Frank, dessen Vergesslichkeit sich vor allem auf die Familie zu beziehen scheint so erkennt er in der Bibliothekarin Jennifer (Susan Sarandon, berühmt geworden durch Dead Man Walking), mit der er sich so gerne unterhält, erst sehr spät seine frühere Frau wieder -, willigt schliesslich doch ein, in ein Heim zu ziehen. Dort ist es nun bereits Usus, dass die Bewohner von ihren Robotern begleitet werden.

Der Film endet mit einem scheinbaren Happyend: Frau, Sohn und Tochter Madison (Liv Tyler) sind mit Frank am Tisch vereint, essen und lachen zusammen, doch der Blick des nun sichtlich gealterten Mannes spricht eine andere Sprache. Die menschliche Beziehung, die er sich ersehnt hat, ist mit den Mitgliedern seiner Familie nicht wirklich möglich. Und der Roboter, der sich zum Partner zu verwandeln schien, hat sich als Teil einer virtuellen Welt erwiesen, die mit einem Knopfdruck verschwinden kann.

Robot \& Frank ist kein Film über beginnende Demenz, sondern über den Eigensinn eines alten Mannes, der sich trotz aller zur Schau gestellten Autonomie (die allerdings immer mehr zerbröckelt) nach Freundschaft sehnt. Auch die beste Pflege ist dafür kein Ersatz.

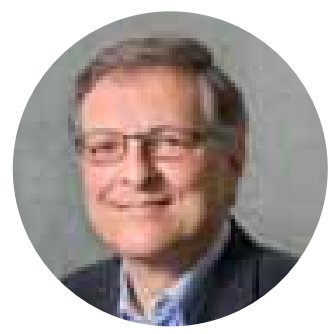

Kurt Seifert ist Leiter Forschung \& Grundlagen bei Pro Senectute Schweiz.

kurt.seifert@pro-senectute.ch 